\title{
Application of Fractional Differential Equations in Modelling the Subdiffusion-Reaction Process
}

\author{
T. Kosztołowicz ${ }^{1 *}$, K. D. Lewandowska ${ }^{2}$ \\ ${ }^{1}$ Institute of Physics, Jan Kochanowski University, \\ ul. Świętokrzyska 15, 25-406 Kielce, Poland \\ ${ }^{2}$ Department of Radiological Informatics and Statistics, Medical University of Gdańsk, \\ ul. Tuwima 15, 80-210 Gdańsk, Poland
}

\begin{abstract}
We focus on a subdiffusion-reaction system in which substances are separated at the initial moment. This system is described by nonlinear differential subdiffusion-reaction equations with a fractional time derivative. These equations are very difficult to solve but there exist methods which allow us to solve them approximately. We discuss how useful such methods are, in particular, the quasistatic approximation method and the perturbation method in analytical solving subdiffusion-reaction equations.
\end{abstract}

Keywords and phrases: nonlinear fractional differential equations, numerical calculations, anomalous diffusion

Mathematics Subject Classification: 35R11, 35K57, 82B80

\section{Introduction}

Subdiffusion is a transport process occurring in systems in which the mobility of particles is significantly hindered due to the internal structure of the medium such as porous media or gels [1-4]. The simplest model of subdiffusion assumes that a subdiffusive particle waits an anomalously long time to take its next step and the mean value of this time is infinite. Subdiffusion is characterized by the mean square displacement of a Brownian particle which is a power function of time $[1,2]$

$$
\left\langle\Delta x^{2}(t)\right\rangle=\frac{2 D_{\alpha}}{\Gamma(1+\alpha)} t^{\alpha},
$$

where $D_{\alpha}$ is the subdiffusion coefficient measured in the units of $\mathrm{m}^{2} / \mathrm{s}^{\alpha}$ and $\alpha$ is a subdiffusion parameter which obeys $0<\alpha<1$ (for normal diffusion there is $\alpha=1$ ).

Subdiffusion is described by a differential equation with the Riemann-Liouville fractional time derivative which can be derived on the basis of continuous time random walk formalism $[1,2]$. This equation reads

$$
\frac{\partial C(x, t)}{\partial t}=D_{\alpha} \frac{\partial^{1-\alpha}}{\partial t^{1-\alpha}} \frac{\partial^{2} C(x, t)}{\partial x^{2}}
$$

\footnotetext{
*Corresponding author. E-mail: tadeusz.kosztolowicz@ujk.edu.pl
} 
where $C$ denotes a concentration of transported substance and the Riemann-Liouville derivative is defined as for $\alpha>0$

$$
\frac{\partial^{\alpha} f(x, t)}{\partial t^{\alpha}}=\frac{1}{\Gamma(j-\alpha)} \frac{\partial^{j}}{\partial t^{j}} \int_{0}^{t} d t^{\prime} \frac{f\left(x, t^{\prime}\right)}{\left(t-t^{\prime}\right)^{1+\alpha-j}},
$$

where $j$ is the smallest natural number which fulfils $j-\alpha>0$.

Let us assume that subdiffusive particles of types $A$ and $B$ can chemically react according to the following formula

$$
m A+n B \rightarrow \emptyset
$$

where $m$ and $n$ are stoichiometric coefficients and $\emptyset$ means that reaction products have no influence on further processes appearing in the system. We also assume that the subdiffusion-reaction process is described by the following equations

$$
\begin{aligned}
& \frac{\partial C_{A}(x, t)}{\partial t}=D_{\alpha A} \frac{\partial^{1-\alpha}}{\partial t^{1-\alpha}} \frac{\partial^{2} C_{A}(x, t)}{\partial x^{2}}-m R_{\alpha}(x, t), \\
& \frac{\partial C_{B}(x, t)}{\partial t}=D_{\alpha B} \frac{\partial^{1-\alpha}}{\partial t^{1-\alpha}} \frac{\partial^{2} C_{B}(x, t)}{\partial x^{2}}-n R_{\alpha}(x, t),
\end{aligned}
$$

where $-\infty<x<\infty$. Following the consideration presented in [5,6], we choose the reaction term, $R_{\alpha}$, in the form

$$
R_{\alpha}(x, t)=\frac{\partial^{1-\alpha}}{\partial t^{1-\alpha}} R(x, t),
$$

where $R(x, t)$ is obtained within the mean-field approximation and reads

$$
R(x, t)=k C_{A}^{m}(x, t) C_{B}^{n}(x, t),
$$

$k$ is the reaction rate constant. We assume that at the initial moment substances $A$ and $B$ are separated, so the initial conditions are as follows

$$
\begin{aligned}
& C_{A}(x, 0)= \begin{cases}C_{0 A}, & x<0, \\
0, & x>0,\end{cases} \\
& C_{B}(x, 0)= \begin{cases}0, & x<0, \\
C_{0 B}, & x>0\end{cases}
\end{aligned}
$$

Equations (1.5) and (1.6) are nonlinear differential ones with fractional derivatives, and are therefore difficult to solve. As far as we know, the exact solutions to the above equations were only found in a very few special cases. In order to find approximate solutions to subdiffusion-reaction equations one usually makes some assumptions which simplify the problem. We mention here the quasistatic approximation method [7-11], the scaling method [8,9,12-14] and the perturbation method [15-17]. We should add here that in many cases some functions which characterize the system, such as the time evolution of a reaction front et al., are enough to describe the process. Let us consider a subdiffusion-reaction system with the initial conditions (1.9) and (1.10). It was observed that when the process starts the characteristic regions and points appear in the system (see Fig. 1) [7-13,18]. There are

- The diffusion region in which the chemical reactions are negligible, thus the only process occurring in this region is a subdiffusive transport.

- The reaction front, $x_{f}(t)$, which is a point placed within the reaction region. At this point the production of the chemical reaction product is the most intensive. There exist a few definitions of this point but with regard to numerical calculations we have chosen the following definition of the reaction front

$$
x_{f}(t)=\frac{\int x R(x, t) d x}{\int R(x, t) d x} .
$$




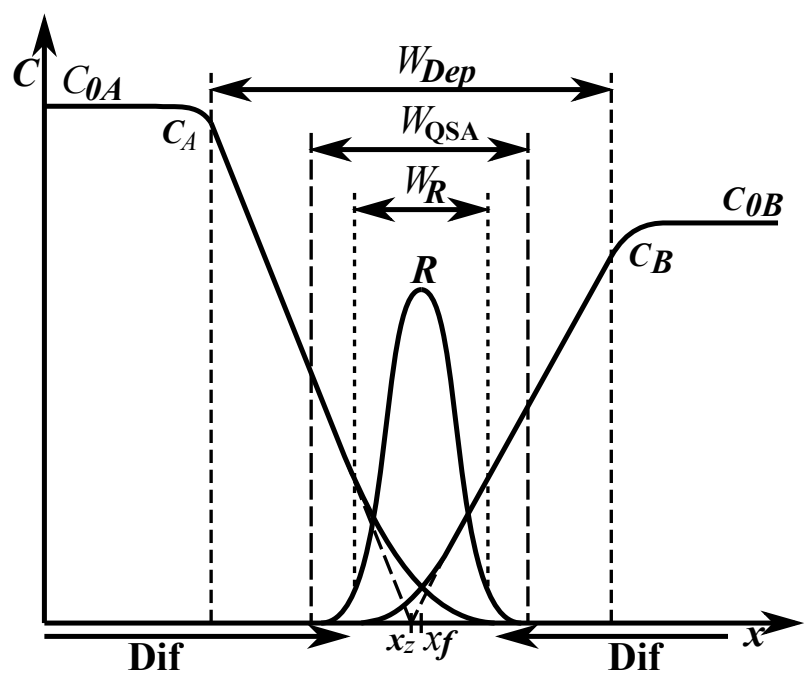

FiguRE 1. Schematic view of the system under consideration; $x_{f}$ is the reaction front, $R$ is the reaction term, 'Dif' is the diffusion region, $W_{\text {Dep }}, W_{\mathrm{R}}$ and $W_{\mathrm{QSA}}$ denote the width of the depletion zone, the reaction region and the quasistatic approximation region, respectively.

- The reaction region in which chemical reactions occur and production of the reaction product is significant. This region is characterized by the reaction region width, $W_{\mathrm{R}}(x, t)$, defined by the following relation [13]

$$
W_{\mathrm{R}}^{2}(x, t)=\frac{\int\left(x-x_{f}(t)\right)^{2} R(x, t) d x}{\int R(x, t) d x} .
$$

- The depletion region which is defined as a region in which the concentrations are significantly smaller than the initial ones.

- The quasistatic approximation region in which the quasistatic approximation method can be used. The definition of this region will be presented later on in this paper.

The existance of these regions and points was confirmed by the numerical results (see, for example, [7]).

In this paper we will present the exact solutions to subdiffusion-reaction equations in the diffusion region and the time evolution of the reaction front obtained by means of the quasistatic approximation method. We will also find the approximate solutions to Eqs. (1.5) and (1.6) by means of the perturbation method. Finally, we will compare our analytical results with numerical solutions.

\section{Numerical method}

The presence of the double derivative (space and time) on the right hand side of Eqs. (1.5) and (1.6) is inconvenient for numerical calculations. Therefore, we rewrite these equations to their equivalent forms $[7,19]$

$$
\begin{aligned}
& \frac{{ }^{C} \partial^{\alpha} C_{A}(x, t)}{\partial t^{\alpha}}=D_{\alpha A} \frac{\partial^{2} C_{A}(x, t)}{\partial x^{2}}-m R(x, t), \\
& \frac{{ }^{C} \partial^{\alpha} C_{B}(x, t)}{\partial t^{\alpha}}=D_{\alpha B} \frac{\partial^{2} C_{B}(x, t)}{\partial x^{2}}-n R(x, t),
\end{aligned}
$$

where ${ }^{C} \partial^{\alpha} f(x, t) / \partial t^{\alpha}$ denotes the Caputo fractional time space derivative which is defined as

$$
\frac{{ }^{C} \partial^{\alpha} f(x, t)}{\partial t^{\alpha}}=\frac{1}{\Gamma(j-\alpha)} \int_{0}^{t} d t^{\prime} \frac{f^{(j)}\left(x, t^{\prime}\right)}{\left(t-t^{\prime}\right)^{1+\alpha-j}},
$$


$f^{(j)}$ denotes the derivative of natural order $j$ and $j-1<\alpha \leq j$. We should add here that the existance of the Caputo fractional derivative on the l.h.s. of Eqs. (2.1) and (2.2) and the Riemann-Liouville fractional derivative on the r.h.s of these equations directly results from continuous time random walk formalism. Next, we use the following relations between fractional derivatives [20]

$$
\frac{\partial^{\alpha} f(x, t)}{\partial t^{\alpha}}=\frac{{ }^{C} \partial^{\alpha} f(x, t)}{\partial t^{\alpha}}+\sum_{i=0}^{j-1} \Phi_{i-\alpha+1}(t) f^{(i)}(x, 0)
$$

where

$$
\Phi_{q+1}(t)=\left\{\begin{array}{cl}
\frac{t^{q}}{\Gamma(q+1)}, & t>0 \\
0, & t \leq 0
\end{array}\right.
$$

and

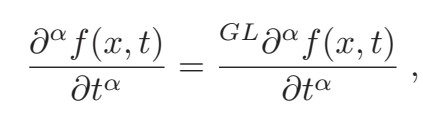

where ${ }^{G L} \partial^{\alpha} f(x, t) / \partial t^{\alpha}$ denotes the Grünwald-Letnikov fractional derivative defined as a limit of a fractional-order backward difference which reads for $\alpha>0$

$$
\frac{G L \partial^{\alpha} f(x, t)}{\partial t^{\alpha}}=\lim _{\Delta t \rightarrow 0}(\Delta t)^{-\alpha} \sum_{r=0}^{\left[\frac{t}{\Delta t}\right]}(-1)^{r}\left(\begin{array}{c}
\alpha \\
r
\end{array}\right) f(x, t-r \Delta t),
$$

$[x]$ means the integer part of $x$ and

$$
\left(\begin{array}{l}
\alpha \\
r
\end{array}\right)=\frac{\Gamma(\alpha+1)}{r ! \Gamma(\alpha-r+1)}=\frac{\alpha(\alpha-1)(\alpha-2) \cdot \ldots \cdot[\alpha-(r-1)]}{1 \cdot 2 \cdot 3 \cdot \ldots \cdot r} .
$$

Using the relations (2.4), (2.6) and (2.7) we can express the Caputo fractional derivative in terms of the fractional-order backward difference

$$
\frac{{ }^{C} \partial^{\alpha} f(x, t)}{\partial t^{\alpha}}=\lim _{\Delta t \rightarrow 0}(\Delta t)^{-\alpha} \sum_{r=0}^{\left[\frac{t}{\Delta t}\right]}(-1)^{r}\left(\begin{array}{l}
\alpha \\
r
\end{array}\right) f(x, t-r \Delta t)-\frac{1}{t^{\alpha} \Gamma(1-\alpha)} f(x, 0) .
$$

In order to obtain a formula useful in numerical calculation we omit the limit in Eq. (2.8) and change the infinite series occurring in Eq. (2.8) to the finite one

$$
\frac{{ }^{C} \partial^{\alpha} f(x, t)}{\partial t^{\alpha}} \simeq(\Delta t)^{-\alpha} \sum_{r=0}^{L}(-1)^{r}\left(\begin{array}{l}
\alpha \\
r
\end{array}\right) f(x, t-r \Delta t)-\frac{1}{t^{\alpha} \Gamma(1-\alpha)} f(x, 0),
$$

where the arbitrary chosen parameter $L$ is called the memory length. We also use the standard approximation of the second order space derivative, namely

$$
\frac{\partial^{2} f(x, t)}{\partial x^{2}} \simeq \frac{f(x+\Delta x, t)-2 f(x, t)+f(x-\Delta x, t)}{(\Delta x)^{2}} .
$$

Substituting Eqs. (2.9) and (2.10) into Eqs. (2.1) and (2.2) we obtain the following algorithm

$$
\begin{aligned}
C_{i}(x, t)= & -\sum_{r=1}^{L}(-1)^{r} \frac{\alpha(\alpha-1)(\alpha-2) \cdot \ldots \cdot[\alpha-(r-1)]}{1 \cdot 2 \cdot 3 \cdot \ldots \cdot r} C_{i}(x, t-r \Delta t)+\frac{(\Delta t)^{\alpha}}{t^{\alpha} \Gamma(1-\alpha)} C_{i}(x, 0) \\
& +D_{\alpha i} \frac{(\Delta t)^{\alpha}}{(\Delta x)^{2}}\left[C_{i}(x+\Delta x, t-\Delta t)-2 C_{i}(x, t-\Delta t)+C_{i}(x-\Delta x, t-\Delta t)\right] \\
& -d_{i} k(\Delta t)^{\alpha} C_{A}^{m}(x, t-\Delta t) C_{B}^{n}(x, t-\Delta t),
\end{aligned}
$$


where $i=A, B, d_{A}=m$ and $d_{B}=n$.

We have omitted a detailed analysis of the stability of the numerical algorithm and its convergence. We have only noticed that the procedure of the numerical solving of fractional subdiffusion equations is stable if a combination of arguments and parameters (here $x / \sqrt{D_{\alpha} t^{\alpha}}$ ) is less then 0.67 (see, for example, $[7,19]$ and references cited therein).

Subdiffusion is a process with a time memory. This arises a problem with the memory length $L$. Podlubny [20] proposed the short memory principle. According to this principle if the memory length is relatively small (and equals about $10 \%$ of the actual number of time steps), the accuracy of the concentrations calculated numerically will be satisfactory even over sufficiently long times. However, in the paper [19] it was shown that this principle is not suffcient for the subdiffusion case in the system with the initial condition (1.9) and (1.10). A satisfactory agreement between numerical results and analytical results can be obtained when the memory length equals at least $80 \%$ of the actual number of time steps.

\section{Solutions obtained by using the quasistationary approximation method}

\subsection{Solutions within the diffusion region}

The diffusion region is the part of the system under consideration, in which almost pure subdiffusion occurs. Therefore, the subdiffusion-reaction equations (1.5) and (1.6) take the form of the pure subdiffusion ones (i.e. without chemical reactions)

$$
\begin{aligned}
& \frac{\partial C_{A \text { Dif }}(x, t)}{\partial t}=D_{\alpha A} \frac{\partial^{1-\alpha}}{\partial t^{1-\alpha}} \frac{\partial^{2} C_{A \text { Dif }}(x, t)}{\partial x^{2}}, \\
& \frac{\partial C_{B \text { Dif }}(x, t)}{\partial t}=D_{\alpha B} \frac{\partial^{1-\alpha}}{\partial t^{1-\alpha}} \frac{\partial^{2} C_{B \text { Dif }}(x, t)}{\partial x^{2}},
\end{aligned}
$$

where $C_{A \text { Dif }}$ and $C_{B \text { Dif }}$ denote the solutions in the diffusion region. The initial conditions are (1.9) and (1.10) and we propose the following boundary conditions

$$
\begin{array}{cl}
\lim _{x \rightarrow-\infty} C_{A \text { Dif }}(x, t)=C_{0 A}, & \lim _{x \rightarrow+\infty} C_{B \text { Dif }}(x, t)=C_{0 B}, \\
C_{A \text { Dif }}\left(x_{z a}, t\right)=0, & C_{B \text { Dif }}\left(x_{z b}, t\right)=0,
\end{array}
$$

where $x_{z a}$ and $x_{z b}$ are some points at which the extensions of the concnetrations $C_{A \text { Dif }}$ and $C_{B \text { Dif }}$ reach the zero value. These boundary conditions are equivalent to the assumption that fully absorbing walls occur at these points. Such points certainly exist because outside the diffusion region at least one of the concentrations $C_{A \text { Dif }}$ or $C_{B \text { Dif }}$ has to equal zero since, in the opposite case, the reaction term would be non-zero. Furthermore, the existance of such points is confirmed by numrical calculations. Since $x_{z a, z b}$ evolve over time [7], the boundary conditions (3.4) depend on time. This fact creates problems when solving differential equations with moving boundary conditions [21]. To simplify the problem we will choose the boundary conditions in a different way. That is to say, we assume that the transport of substances within the diffusion region is similar to the transport of substances in a subdiffusive system with a partially absorbing wall — placed at the initial point - at which substances in the system under consideration are separated $(x=0)$. Then, we postulate the following Green functions in the diffusion region

$$
\begin{aligned}
& G_{A \text { Dif }}\left(x, t ; x_{0}\right)=G_{0 A}\left(x, t ; x_{0}\right)-\sigma_{A} G_{0 A}\left(x, t ;-x_{0}\right), \\
& G_{B \text { Dif }}\left(x, t ; x_{0}\right)=G_{0 B}\left(x, t ; x_{0}\right)-\sigma_{B} G_{0 B}\left(x, t ;-x_{0}\right),
\end{aligned}
$$

where $G_{0 A}$ and $G_{0 B}$ are Green functions for unbounded systems without chemical reactions, which read

$$
G_{0 i}\left(x, t ; x_{0}\right)=\frac{1}{\alpha\left|x-x_{0}\right|} H_{11}^{10}\left(\left(\frac{\left|x-x_{0}\right|}{\sqrt{D_{\alpha i} t^{\alpha}}}\right)^{2 / \alpha} \mid \begin{array}{ll}
1 & 1 \\
1 & 2 / \alpha
\end{array}\right),
$$


$i=A, B, \sigma_{i}$ are parameters which determine the absorbing properties of the wall and $H$ denotes the Fox function, which can be expressed as the series [22]

$$
H_{11}^{10}\left(u \mid \begin{array}{ll}
1 & 1 \\
p & q
\end{array}\right)=\frac{1}{q} u^{p / q} \sum_{j=0}^{\infty} \frac{(-1)^{j}}{j ! \Gamma(1-p / q-j / q)} u^{j / q} .
$$

The physical meaning of (3.5) and (3.6) was briefly discussed in [23]. Using the integral formulae

$$
\begin{aligned}
& C_{A \text { Dif }}(x, t)=C_{0 A} \int_{-\infty}^{0} d x_{0} G_{A \text { Dif }}\left(x, t ; x_{0}\right), \\
& C_{B \text { Dif }}(x, t)=C_{0 B} \int_{0}^{\infty} d x_{0} G_{B \text { Dif }}\left(x, t ; x_{0}\right),
\end{aligned}
$$

we find (for details of calculations see [7])

$$
C_{A \mathrm{Dif}}(x, t)=C_{0 A}-\frac{2}{\alpha} \eta_{A} H_{11}^{10}\left(\left(\frac{-x}{\sqrt{D_{\alpha A} t^{\alpha}}}\right)^{2 / \alpha} \mid \begin{array}{ll}
1 & 1 \\
0 & 2 / \alpha
\end{array}\right),
$$

where

$$
\eta_{A}=C_{0 A}\left(1+\sigma_{A}\right) / 2
$$

and

$$
C_{B \text { Dif }}(x, t)=C_{0 B}-\frac{2}{\alpha} \eta_{B} H_{11}^{10}\left(\left(\frac{x}{\sqrt{D_{\alpha B} t^{\alpha}}}\right)^{2 / \alpha} \mid \begin{array}{ll}
1 & 1 \\
0 & 2 / \alpha
\end{array}\right)
$$

where

$$
\eta_{B}=C_{0 B}\left(1+\sigma_{B}\right) / 2 .
$$

In Fig. 2 we present the comparison between the analytical solutions obtained within the diffusion region and the numerical ones. The agreement within the diffusion region is quite satisfactory.

\subsection{The quasistationary approximation method}

The quasistatic approximation is based on the assumption that concentrations are slowly varying functions of time in the quasistatic approximation region (see Fig. 1). Consequently, a time derivative on the righthande side of the subdiffusion-reaction equations (1.5) and (1.6) is small and can be neglected in this region, thus these equations take the form

$$
\begin{aligned}
\frac{\partial^{1-\alpha}}{\partial t^{1-\alpha}}\left[D_{\alpha A} \frac{\partial^{2} C_{A}(x, t)}{\partial x^{2}}-m R(x, t)\right] & =0, \\
\frac{\partial^{1-\alpha}}{\partial t^{1-\alpha}}\left[D_{\alpha B} \frac{\partial^{2} C_{B}(x, t)}{\partial x^{2}}-n R(x, t)\right] & =0 .
\end{aligned}
$$

Within the diffusion region in which the reaction term is negligible, the quasistatic approximation method can be used when the concentrations are linear functions of $x$. The borders of the quasistatic approximation region are placed ouside the reaction region and inside the diffusion one, in the parts in which the concentration is a linear function of $x$. Let us note that the quasistatic approximation region contains the whole reaction region and partially overlaps the diffusion ones. In this overlapping region we will join the solution obtained in the quasistatic approximation region and in the diffuson ones. Since the points $x_{z}$ and $x_{f}$ are placed very close to each other we can identify them. Finally, we obtain the time evolution of the reaction front which reads

$$
x_{f}(t)=K t^{\alpha / 2}
$$




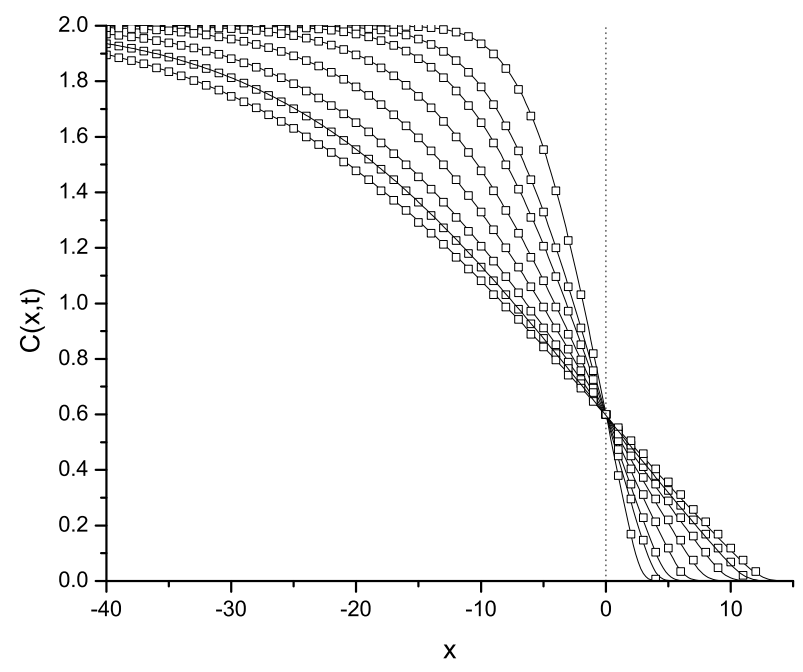

Figure 2. Concentration profiles obtained numerically (squares) and the analytical solution (3.11) (solid lines). Calculations were performed for $D_{\alpha A}=0.025, D_{\alpha B}=0.0125$, $C_{0 A}=2, C_{0 B}=1, m=n=1, k=1, \eta_{A}=0.6$ and for the following times $0.5,1,1.5$, $2.5,4,6,8,10\left(\times 10^{3}\right)($ curves which are placed closer to the $x$-axis in the left part of the graph correspond to longer times). All quantities are given in arbitrarily chosen units.

where the coefficient $K$ is the solution to the following equation

$$
\Phi\left(\frac{-K}{\sqrt{D_{\alpha A}}}\right)=\frac{n}{m} \frac{\sqrt{D_{\alpha A}} C_{0 A}}{\sqrt{D_{\alpha B}} C_{0 B}} \Phi\left(\frac{K}{\sqrt{D_{\alpha B}}}\right) .
$$

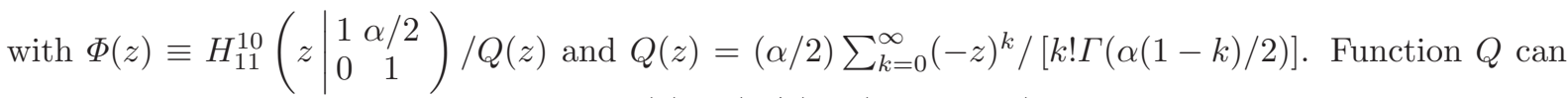
also be presented as the Wright function $Q(z)=(\alpha / 2) W\left(-z ;-\frac{\alpha}{2}, \frac{\alpha}{2}\right)$. The time evolution of the reaction front is the power function with the exponent depending on the subdiffusion parameter $\alpha$ alone, while the subdiffusion coefficients $D_{\alpha A}$ and $D_{\alpha B}$ control the parameter $K$.

\section{The perturbation method}

Within the perturbation method one assumes that a nonlinear term occurring in the subdiffusion-reaction equation depends on a small parameter $\varepsilon$, and the solutions are in the form of a power series with respect to the parameter $\varepsilon$. Below we check if the perturbation method up to the first order is effective in solving subdiffusion-reaction equations. To check this we choose a symmetrical system in which $m=n=1$, $C_{0 A}=C_{0 B} \equiv C_{0}$ and $D_{\alpha A}=D_{\alpha B} \equiv D$. The symmetry of the system ensures $C_{A}(x, t)=C_{B}(-x, t)$ and that the reaction front does not change its position. In order to solve Eqs. (2.1) and (2.2) by means of the perturbation method, we firstly transform these equations into a dimensionless form using the substitutions:

$$
x=\rho x_{s}, \quad t=\tau t_{s},
$$


where $\rho$ and $\tau$ denote the dimensionless position and time, $x_{\mathrm{s}}$ and $t_{\mathrm{s}}$ are some constants of the space and time dimension, respectively. After calculations we obtain

$$
\begin{aligned}
& \frac{{ }^{C} \partial^{\alpha} a(\rho, \tau)}{\partial \tau^{\alpha}}=\frac{\partial^{2} a(\rho, \tau)}{\partial \rho^{2}}-\varepsilon a(\rho, \tau) b(\rho, \tau), \\
& \frac{{ }^{C} \partial^{\alpha} b(\rho, \tau)}{\partial \tau^{\alpha}}=\frac{\partial^{2} b(\rho, \tau)}{\partial \rho^{2}}-\varepsilon a(\rho, \tau) b(\rho, \tau),
\end{aligned}
$$

where

$$
a(\rho, \tau)=\frac{C_{A}\left(\rho x_{s}, \tau t_{s}\right)}{C_{0}}, \quad b(\rho, \tau)=\frac{C_{B}\left(\rho x_{s}, \tau t_{s}\right)}{C_{0}}, \quad x_{s}=\sqrt{D t_{s}^{\alpha}}, \quad \varepsilon=k t_{s}^{\alpha} C_{0} .
$$

Next, we assume that the solutions to Eqs. (4.2) and (4.3) are the power series with respect to the parameter $\varepsilon$ which is assumed to be small $(\varepsilon \ll 1)$

$$
a(\rho, \tau)=\sum_{n=0}^{\infty} \varepsilon^{n} a_{n}(\rho, \tau), \quad b(\rho, \tau)=\sum_{n=0}^{\infty} \varepsilon^{n} b_{n}(\rho, \tau) .
$$

Substituting Eq. (4.5) to Eqs. (4.2) and (4.3) and comparing functions of the same order with respect to the parameter $\varepsilon$ occurring on both sides of these equations, we get equations for the functions of $n$-th order $(n \geq 0)$

$$
\begin{aligned}
& \frac{{ }^{C} \partial^{\alpha} a_{n}(\rho, \tau)}{\partial \tau^{\alpha}}=\frac{\partial^{2} a_{n}(\rho, \tau)}{\partial \rho^{2}}-R_{n}(\rho, \tau), \\
& \frac{{ }^{C} \partial^{\alpha} b_{n}(\rho, \tau)}{\partial \tau^{\alpha}}=\frac{\partial^{2} b_{n}(\rho, \tau)}{\partial \rho^{2}}-R_{n}(\rho, \tau),
\end{aligned}
$$

where

$$
R_{0}(\rho, \tau) \equiv 0, \quad R_{n}(\rho, \tau)=\sum_{k=0}^{n-1} a_{k}(\rho, \tau) b_{n-k-1}(\rho, \tau), \quad n \geq 1
$$

with the initial condition

$$
\begin{gathered}
a_{0}(\rho, 0)=\Theta(-\rho), \quad b_{0}(\rho, 0)=\Theta(\rho), \\
a_{n}(\rho, 0) \equiv b_{n}(\rho, 0) \equiv 0, \quad n \geq 1,
\end{gathered}
$$

where $\Theta$ is the Heaviside function and with the boundary conditions

$$
\begin{gathered}
a_{0}(-\infty, \tau)=b_{0}(+\infty, \tau)=1, \quad a_{0}(+\infty, \tau)=b_{0}(-\infty, \tau)=0, \\
a_{n}(-\infty, \tau)=a_{n}(+\infty, \tau)=0, \quad b_{n}(-\infty, \tau)=b_{n}(+\infty, \tau)=0, \quad n \geq 1 .
\end{gathered}
$$

The reaction term disappears for zeroth order equations $(n=0)$ so these equations appear to be pure subdiffusion equations without chemical reactions. We note that solutions to these equations are well known $[1,2,22]$. In order to solve equations of higher order $(n>0)$ we will use the Laplace transform method. In practice, it is extremaly difficult to solve equations of an order higher than one due to their complicated forms. Therefore, we assume that the approximate solutions are

$$
a=a_{0}+\varepsilon a_{1}, \quad b=b_{0}+\varepsilon b_{1} .
$$

As we mentioned above, the zeroth order solutions correspond with the pure subdiffusion process. Chemical reactions reduce the amount of particles, thus solutions of the first order are expected to be negative. If they are not for some $\rho$, we put $a_{1}(\rho, \tau)=b_{1}(\rho, \tau)=0$. Finally, we return the obtained 
solutions to the dimensional form. After calculations we find the dimensional zeroth order solutions which read

$$
\begin{aligned}
& C_{A 0}^{-}(x, t)=C_{0}\left[1-\frac{1}{2} \sum_{i=0}^{\infty} \frac{1}{\Gamma(1-\alpha i / 2) i !}\left(\frac{x}{\sqrt{D} t^{\alpha / 2}}\right)^{i}\right], \\
& C_{A 0}^{+}(x, t)=\frac{C_{0}}{2} \sum_{i=0}^{\infty} \frac{1}{\Gamma(1-\alpha i / 2) i !}\left(-\frac{x}{\sqrt{D} t^{\alpha / 2}}\right)^{i},
\end{aligned}
$$

where - and + denotes the solution for $x<0$ and $x>0$, respectively. Functions (4.14) and (4.15) can also be presented as the Wright functions $C_{A 0}^{-}(x, t)=C_{0}-\left(C_{0} / 2\right) W\left(\frac{x}{\sqrt{D_{\alpha} t^{\alpha}}} ;-\frac{\alpha}{2}, 1\right)$ and $C_{A 0}^{+}(x, t)=$ $\left(C_{0} / 2\right) W\left(-\frac{x}{\sqrt{D_{\alpha} t^{\alpha}}} ;-\frac{\alpha}{2}, 1\right)$.

The dimensional solutions of the first order are [24]

$$
\begin{aligned}
& C_{A 1}^{-}(x, t)=C_{0}^{2} k t^{\alpha}\left[-\frac{1}{4} \sum_{i=0}^{\infty} Q_{i}\left(\frac{x^{2}}{D t^{\alpha}}\right)^{i}+\sum_{i=1}^{\infty} P_{i}^{-}\left(\frac{x}{\sqrt{D} t^{\alpha / 2}}\right)^{i}\right], \\
& C_{A 1}^{+}(x, t)=C_{0}^{2} k t^{\alpha}\left[-\frac{1}{4} \sum_{i=0}^{\infty} Q_{i}\left(\frac{x^{2}}{D t^{\alpha}}\right)^{i}+\sum_{i=1}^{\infty} P_{i}^{+}\left(\frac{x}{\sqrt{D} t^{\alpha / 2}}\right)^{i}\right],
\end{aligned}
$$

where $Q_{i}$ and $P_{i}^{\mp}$ are defined as

$$
\begin{aligned}
Q_{i} & =\frac{1}{(2 i) ! \Gamma(1-(i-1) \alpha)}, \\
P_{i}^{-} & =\frac{1}{(i) ! \Gamma(1-\alpha(i / 2-1))} \sum_{m=0}^{[(i+1) / 2]-1}(m) ! e_{m}, \\
P_{i}^{+} & =\frac{1}{(i) ! \Gamma(1-\alpha(i / 2-1))} \sum_{m=0}^{[(i+1) / 2]-1}(m) ! e_{m}^{\prime},
\end{aligned}
$$

with $e_{0}=e_{0}^{\prime}=1 / 4, e_{1}=e_{1}^{\prime}=0$, and

$$
\begin{aligned}
& e_{k}=\frac{1}{4 k !}\left[1-\sum_{j=1}^{k} \frac{\Gamma(1-\alpha k / 2) k !}{\Gamma(1-\alpha j / 2) j ! \Gamma(1-\alpha(k-j) / 2)(k-j) !}\right], \\
& e_{k}^{\prime}=\frac{(-1)^{k}}{2 k !}\left[1-\frac{1}{2} \sum_{j=1}^{k} \frac{\Gamma(1-\alpha k / 2) k !}{\Gamma(1-\alpha j / 2) j ! \Gamma(1-\alpha(k-j) / 2)(k-j) !}\right],
\end{aligned}
$$

$k \geq 2,[u]$ denotes an integer part of a number $u([u] \leq u)$.

Finally, the approximate solutions to Eqs. (2.1) and (2.2) read

$$
\begin{aligned}
& C_{A}^{ \pm}(x, t)=C_{A 0}^{ \pm}(x, t)+C_{A 1}^{ \pm}(x, t), \\
& C_{B}^{ \pm}(x, t)=C_{A}^{ \pm}(-x, t) .
\end{aligned}
$$

In Fig. 3 we present a comparison between analytical and numerical solutions. We observe that agreement between these solutions is reasonably accurate for the times presented in the figure. The numerical solutions can be treated as the basis for comparison with other approximate results because, as it was indicated in $[7,19]$ they are in satisfactory accordance with the analytical results. Therefore, if we compare the analytical solutions with the numerical ones (see Fig. 3) we can observe that the accuracy of analytical solutions decreases as time increases. 


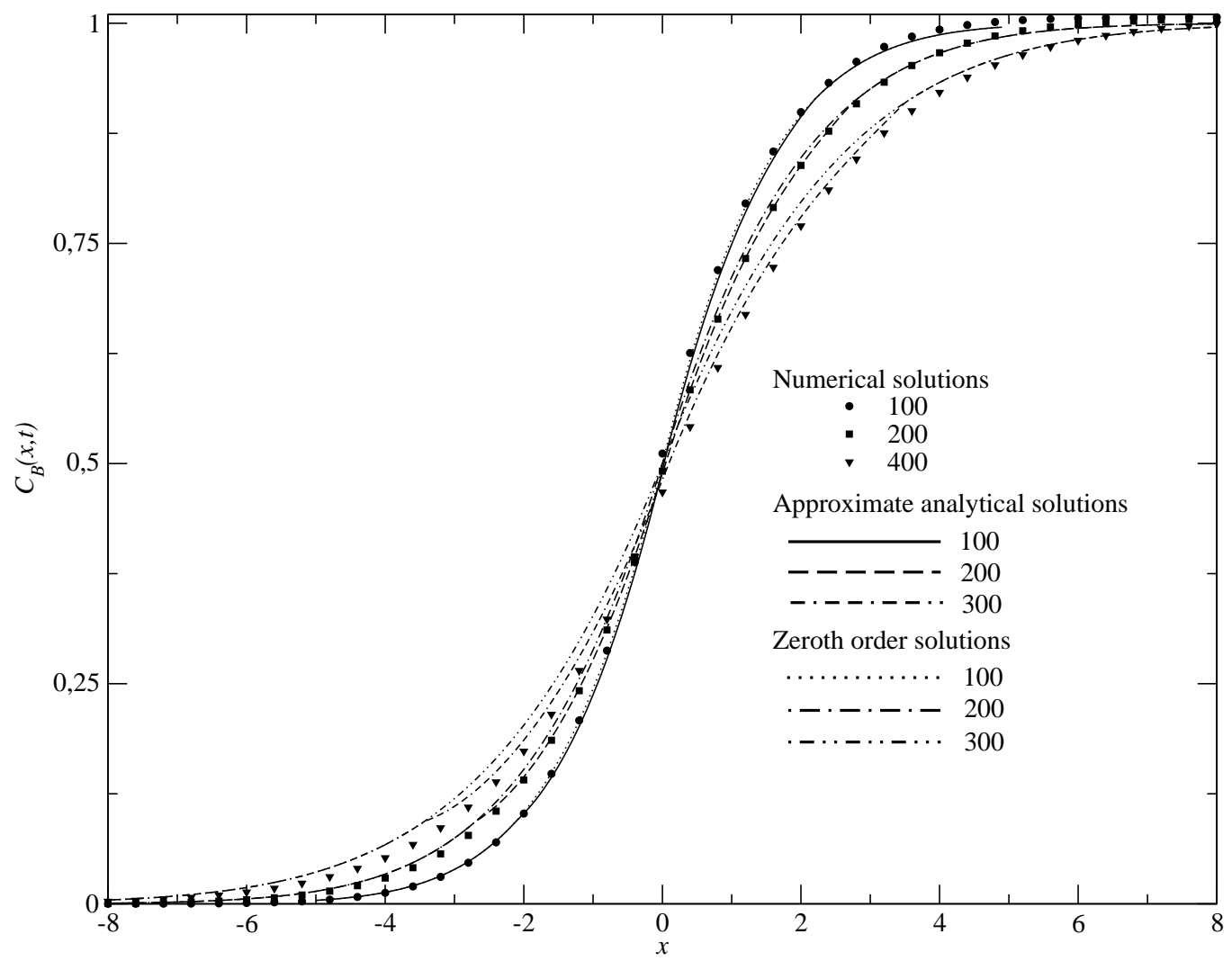

Figure 3. Concentration profiles calculated for $\alpha=0.7, D=0.05, C_{0}=1, k=0.001$ and for the times given in the legend; symbols represent the numerical solutions, lines the approximate analytical solutions (4.24) and the zeroth order solutions (i.e. for the system without chemical reactions).

\section{Final remarks}

In this paper we have considered the subdiffusion-reaction system in which the substances $A$ and $B$ are separated at the initial moment (Eqs. (1.9) and (1.10)). This system is described by nonlinear differential subdiffusion-reaction equations with fractional time derivatives (1.5) and (1.6). Since such equations are difficult to solve, we have found, and by means of the perturbation method, the approximate concentration profiles (4.23) and (4.24), and, by means of the quasistationary approximation method, we have shown that the time evolution of the reaction front for the subdiffusion-reaction system with the initial condition (1.9) and (1.10) is a power function of time (3.17).

Using subdiffusion-reaction equations in describing carious-lesion progress in human tooth-enamel (one of the substances is then static), we found that in some cases a time evolution of the carious-lesion depth (which is identified with the reaction front) [25], evolves over time according to the formula

$$
x_{f} \sim t^{\alpha / 2} .
$$

Thus, we conclude that inside human tooth-enamel subdiffusion of organic acids can occur.

We should add here that the perturbation method was used to solve normal diffusion-reaction equations in the case of one static reactant. The obtained solution was sucesfully used to model carious-lesion progress in a human tooth [15]. It could be said that in this case the perturbation method showed 
itself effective. Thus, we expect that this method will also be effective in modelling subdiffusion-reaction processes with one static reactant.

Acknowledgements. This paper was partially supported by the Polish National Science Centre under grant No. N N202 195640 (1956/B/H03/2011/40).

\section{References}

[1] R. Metzler, J. Klafter. The random walk's guide to anomalous diffusion: a fractional dynamics approach. Phys. Rep., 339 (2000), 1-77.

[2] R. Metzler, J. Klafter. The restaurant at the end of the random walk: recent developments in the description of anomalous transport by fractional dynamics. J. Phys., A 37 (2004), R161-R208.

[3] T. Kosztołowicz, K. Dworecki, S. Mrówczyński. How to measure subdiffusion parameters. Phys. Rev. Lett., 94 (2005), 170602-1-170602-4.

[4] T. Kosztołowicz, K. Dworecki, S. Mrówczyński. Measuring subdiffusion parameters. Phys. Rev., E 71 (2005), 0411051-041105-11.

[5] K. Seki, M. Wojcik, M. Tachiya. Recombination kinetics in subdiffusive media. J. Chem. Phys., 119 (2003), $7525-7533$.

[6] S.B. Yuste, L. Acedo, K. Lindenberg. Reaction front in an $A+B \rightarrow C$ reaction-subdiffusion process. Phys. Rev., E 69 (2004), 036126-1-036126-10.

[7] T. Kosztołowicz, K.D. Lewandowska. Time evolution of the reaction fornt in a subdiffusive system. Phys. Rev., E 78 (2008), 066103-1-066103-11.

[8] D. ben-Avraham, S. Havlin. Diffusion and Reactions in Fractals and Disordered Systems. Cambridge University Press, Cambridge, 2000.

[9] V. Méndez, S. Fedotov, W. Horsthemke. Reaction-Transport Systems: Mesoscopic Foundations, Fronts, and Spatial Instabilities. Springer-Verlag, Berlin, 2010.

[10] Z. Koza. The long-time behavior of initially separated $A+B \rightarrow 0$ reaction-diffusion systems with arbitrary diffusion constants. J. Stat. Phys., 85 (1996), 179-191.

[11] Z. Koza. The long-time behaviour of initially separated $A+B($ static $) \rightarrow 0$ reaction-diffusion systems. Physica, A 240 (1997), 622-634.

[12] M.Z. Bazant, H.A. Stone. Asymptotics of reaction-diffusion fronts with one static and one diffusing reactant. Physica, D 147 (2000), 95-121.

[13] L. Gálfi, Z. Rácz. Properties of the reaction front in an $A+B \rightarrow C$ type reaction-diffusion process. Phys. Rev., A 38 (1988), 3151-3154.

[14] S. Cornell, S. Koza, M. Droz. Dynamic Multiscaling of the Reaction-Diffusion Front for $m A+n B \rightarrow 0$. Phys. Rev., E 52 (1995), 3500-3505.

[15] K.D. Lewandowska, T. Kosztołowicz. Application of diffusion-reaction equations to model carious lesion progress. Physica, A 391 (2012), 2608-2616.

[16] H. Taitelbaum, Y-E.L. Koo, S. Havlin, R. Kopelman, G.H. Weiss. Exotic Behavior of the Reaction Front in the $A+B \rightarrow C$ Reaction-Diffusion System. Phys. Rev., A 46 (1992), 2151-2154.

[17] H. Taitelbaum, A. Yen, R. Kopelman, S. Havlin, G.H. Weiss. Effects of bias on the kinetics of $A+B \rightarrow C$ with initially separated reactants. Phys. Rev., E 54 (1996), 5942-5947.

[18] Z. Jiang, C. Ebner. Simulation study of reaction fronts. Phys. Rev., A 42 (1990), 7483-7486.

[19] K.D. Lewandowska, T. Kosztołowicz. Numerical study of subdiffusion equation. Acta Phys. Pol., B 38 (2007), 18471854.

[20] I. Podlubny. Fractional differential equations. Academic Press, San Diego, 1999

[21] J. Crank. The mathematics of diffusion. Clarendon Press, Oxford, 1975

[22] T. Kosztołowicz. From solutions of diffusive equation to the solution of subdiffusive one. J. Phys., A 37 (2004), 1077910789

[23] T. Kosztołowicz, K. Dworecki, K.D. Lewandowska. Subdiffusion in a system with thin membranes. Phys. Rev., E 86 (2012), 021123-1-021123-7.

[24] K.D. Lewandowska, T. Kosztołowicz, M. Piwnik. The perturbation method to solve subdiffudion-reaction equations. Acta Phys. Pol., B 43 (2012), 1065-1071.

[25] K.D. Lewandowska, T. Kosztołowicz. Time evolution of the reaction front in a subdiffusive system. In: Noise and fluctuations, 2007, edited by M. Tacano, Y. Yamamoto, M. Nakao. American Institute of Physics, Melville, 2007. 